\title{
Clinico-Pathological Study of Skin Epidermal and Appendageal Tumors
}

\author{
Dr. Balaji Govindan MD DVL \\ Senior Assistant Professor, Department of Dermato-Venereology, Govt Mohan Kumaramangalam Medical College Hospital, Salem, \\ Tamilnadu - 636001 , India
}

\begin{abstract}
Aim of the study: To find out the (i) frequency of surface epidermal and appendageal tumors reported in the department of dermatology of a tertiary care hospital, (ii) clinical presentations, and (iii) their histological features. Materials and methods: 60 patients presenting with different types of epidermal and appendages tumors as their main complaints were selected for the study. Skin biopsy and the relevant investigations were done. Results: Out of 60 patients studied, 29 were male $(48.33 \%)$ and 31 were female (51.66\%), most of the tumors $(80 \%)$ occurred after 20 years of age and benign tumors (85\%) outnumbered malignant ones. Syringoma (20\%) was the most frequent benign tumor and basal cell carcinoma (8.33\%), the most common among the malignant tumors. Only one case of epidermal nevus patient had seizures and visual disturbances. Also, rare association of squamous cell carcinoma with xeroderma pigmentosa patient and growth retardation has been documented. Conclusion: The incidence of benign tumors was more when compared to the malignant tumors and all the tumors had their characteristic histological features.
\end{abstract}

Keywords: Skin tumors, Syringoma, Basal cell carcinoma

\section{Introduction}

In recent decades, scientific inquiry have revealed skin as a complex organ in which precisely regulated cellular interactions govern many crucial responses to our environment. Factors affecting the delicate homeostasis that exists among the skin cells results in mere wrinkles to life threatening cancers and disorders of immune regulation [1]. In India, malignant skin tumors (ST) constitute about 1-2\% of all cancers. Various cancer registries in India reported cumulative incidence of ST varying from 0.5 to 2 per 100000 populations [2]. In spite of growing public awareness of harmful effects of sun exposure, incidence of ST continues to rise. ST are associated with substantial morbidity, disfigurement, and their treatment is costly. Early diagnosis can reduce morbidity and cost. This study of tumors of epidermis and appendages has been undertaken to find out the frequency of benign and malignant growths, and their clinic pathological correlation.

\section{Materials and Methods}

60 patients presenting with different forms of epidermal tumors of skin and appendages as their main complaints were selected for the study from the skin department of a tertiary hospital. The provisional diagnosis was mainly made by clinical presentations. The age and sex of all the sixty cases along with their occupation were recorded. The duration of the skin lesions in all the patients was also noted. Specific and relevant histories were taken from certain cases with skin tumors (ST) and they included history of prolonged intake of any internal medication like inorganic arsenic containing preparation which may lead to basal cell epithelioma. Family history regarding the presence of tumors was also elicited and it was relevant in trichoepithelioma and steatocystoma multiplex. History of medical and surgical intervention for the above complaints if any was also noted in all the sixty patients. Thorough clinical examination of the skin lesions was carried out in all the cases with special reference to the site, number, size, shape, color, surface, borders, consistency, tenderness and compressibility of the lesions. Whether the lesions were grouped or discrete, sessile, or pedunculated or whether there were any attachment to the underlying structures or the overlying skin were also observed. Careful systemic examinations were carried out. Investigations like complete hemogram and skin biopsy in the form of both excision and incision biopsy were carried out. The sections for histopathological examination were stained with hematoxylin and eosin. In selected cases, X-ray skull, barium meal study, barium enema, upper gastrointestinal endoscopy, ultrasonogram was carried out. Most of the patients were treated surgically in the form of complete excision of the lesions. Few cases were treated with electrocautery, and cryotherapy.

\section{Results}

Of 60 patients studied, 29 were male $(48.33 \%)$ and 31 were female $(51.66 \%)$ as shown in Chart 1 . The distribution of skin tumors is given in Chart 2. The age and sex distribution is depicted in Table -1 .

Male $=29$

Chart 1: Male - Female Case Distribution

Female $=31$

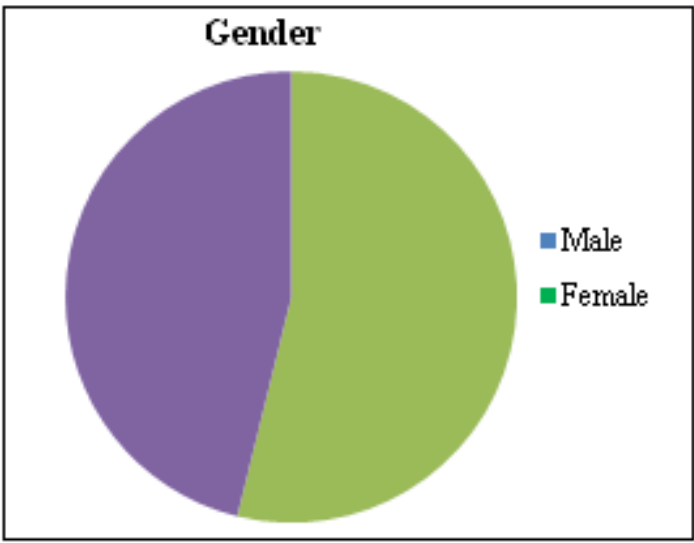




\section{International Journal of Science and Research (IJSR) \\ ISSN (Online): 2319-7064}

Index Copernicus Value (2013): 6.14 | Impact Factor (2014): 5.611

Chart 2: Distribution of tumors as in 60 patients

Syringoma $=12(20 \%)$ Keratoacanthoma $=3(5 \%)$

Seborrheoic Keratosis $=10(16.66 \%)$ Nevus sebaceous $=3(5 \%)$

Epidermal nevus $=9(15 \%)$ Trichoepithelioma $=3(5 \%)$

Epidermal cysts $=9(15 \%)$ Steatocystoma multiplex $=2(3.33 \%)$

Basal cell carcinoma $=5(8.33 \%)$ Sebaceous carcinoma $=1(1.66 \%)$

Squamous cell carcinoma $=3(5 \%)$

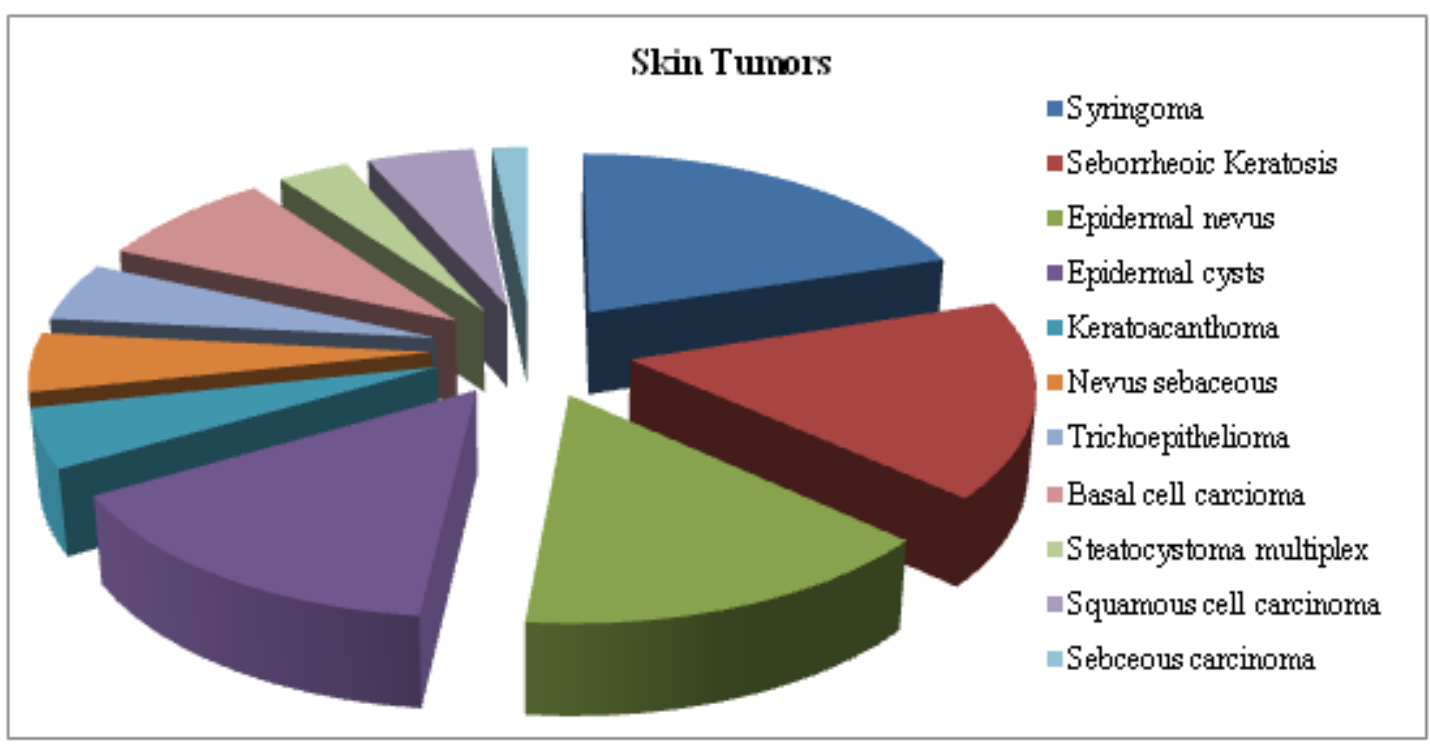

Table 1: Age and Sex Distribution of 60 Skin Tumours

\begin{tabular}{|c|c|c|c|c|}
\hline \multirow[t]{2}{*}{ Age group (yrs) } & \multicolumn{2}{|c|}{ No. of cases } & \multirow[t]{2}{*}{ Total } & \multirow[t]{2}{*}{ Percentage } \\
\hline & Male & Female & & \\
\hline Birth-1 year & 4 & 2 & 6 & 10.00 \\
\hline $2-10$ & 0 & 0 & 0 & 0.00 \\
\hline $11-20$ & 3 & 3 & 6 & 10.00 \\
\hline $21-30$ & 8 & 6 & 14 & 23.33 \\
\hline $31-40$ & 3 & 8 & 11 & 18.33 \\
\hline $41-50$ & 3 & 6 & 9 & 15.00 \\
\hline $51-60$ & 8 & 6 & 14 & 23.33 \\
\hline Total & 29 & 31 & 60 & 100 \\
\hline
\end{tabular}

\section{Syringoma}

Twelve cases of syringoma were observed. All were adults and among them only two were male. The syringoma lesions were skin colored; firm, elevated, flat-topped papules of size ranging from 1 to $5 \mathrm{mms}$ in diameter with angular outline and were distributed mainly over the face especially below the lower eyelids. In one adult female, the lesions were also seen in the neck, in addition to face. All the patients sought medical help only for cosmetic disability. Excision biopsy of a single lesion was done and histopathology with hematoxylin \& eosin stain showed numerous small cystic ductal structures lined by 2 rows of flattened epithelial cells, embedded in dermal fibrous stroma. Some of the ducts also had tail like projections of epithelial stands into the stroma giving a tadpole appearance.

\section{Seborrhoeic Keratosis.}

Among ten cases encountered 6 were male patients and 4 female patients. All the cases occurred over 40 years of age. All the 10 cases were asymptomatic except one female who complained of pruritus over the lesions. She was submitted for investigations to exclude any internal malignancy and that included barium meal, upper gastro-intestinal endoscopy, ultrasonography and occult blood which proved to be negative. Excision biopsy of a lesion was done and histopathology with hematoxylin \& eosin stain showed the classical features of seborrhoeic keratosis.

\section{Epidermal Nevus}

Among the nine cases of epidermal nevus, the lesions were present since birth in 2 male and one female case. Only one of that was linear epidermal nevus. Clinically, the lesions were seen as closely set, keratotic, pigmented verrucous papules in a linear fashion of size $2 \times 1 \mathrm{cms}$ distributed unilaterally over the face, left side of the forehead and left infra orbital region. (Figure 1) Only one patient had seizures and visual disturbances. Subsequent Computerized tomography of brain was normal. Incision biopsy was done and histopathology with hematoxylin \& eosin stain was consistent with epidermal nevus.

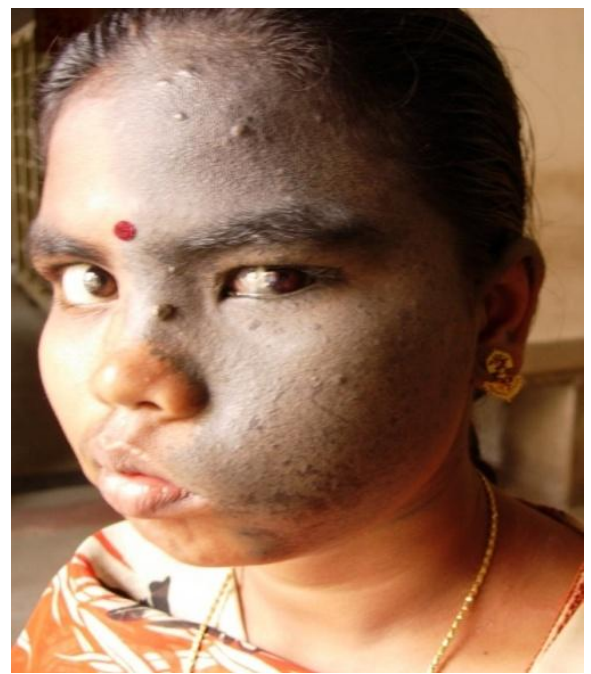

Figure 1: Linear Epidermal Nevus

\section{Volume 5 Issue 2, February 2016}




\section{International Journal of Science and Research (IJSR) \\ ISSN (Online): 2319-7064}

Index Copernicus Value (2013): 6.14 | Impact Factor (2014): 5.611

\section{Epidermal Cyst}

Epidermal cysts were encountered in 5 male patients and 4 female patients. The lesions were asymptomatic in all the cases. These lesions appeared as elevated, rounded, firm, intradermal cystic nodules of 1 to $2 \mathrm{cms}$ in diameter with stretched and shiny surface, found mainly over the scalp and neck with punctum in 4 cases the number of cysts ranged from 1 to 3 and was not attached to the underlying structures. Excision biopsy was done and histopathology with hematoxylin \& eosin stain showed the cyst in the dermis, with cyst wall showing all the layers of true epidermis with lamellated keratin inside the cavity.

\section{Keratoacanthoma}

Two male and one female case were reported. Clinically, presented as dome shaped lesions of size ranging from 1-3 cms in diameter with a central crater. All the three cases were solitary type of keratoacanthomas. Excision biopsy was done and histopathology with hematoxylin \& eosin stain showed central keratin filled crater. The epidermis extended like a buttress over the side of the crater. Also irregular downward proliferations of the epidermis into the dermis and horn cysts were seen.

\section{Nevus Sebaceous Of Jadassohn}

Three adult patients reported with these lesions of whom, 2 were males and 1 female. The lesions in all the 3 patients were present since birth but as smaller and flat ones. The patients also gave history of increase in the size of the lesions with verrucous excrescences since adolescence. Clinically they were seen as circumscribed, hyperkeratotic, thickened, verrucous, plaque of size varying from 2 to $4 \mathrm{cms}$ in diameter and were distributed over the parietal and frontopareital region of the scalp (Figure 2).No other associated eye, skeletal and nervous system involvement. Excision biopsy was done and histopathology with hematoxylin\& eosin staining showed marked hyperkeratosis, irregular acanthosis, papillomatosis, and numerous mature sebaceous glands seen in the upper dermis. The lesions in all the cases were excised with good cosmetic results.

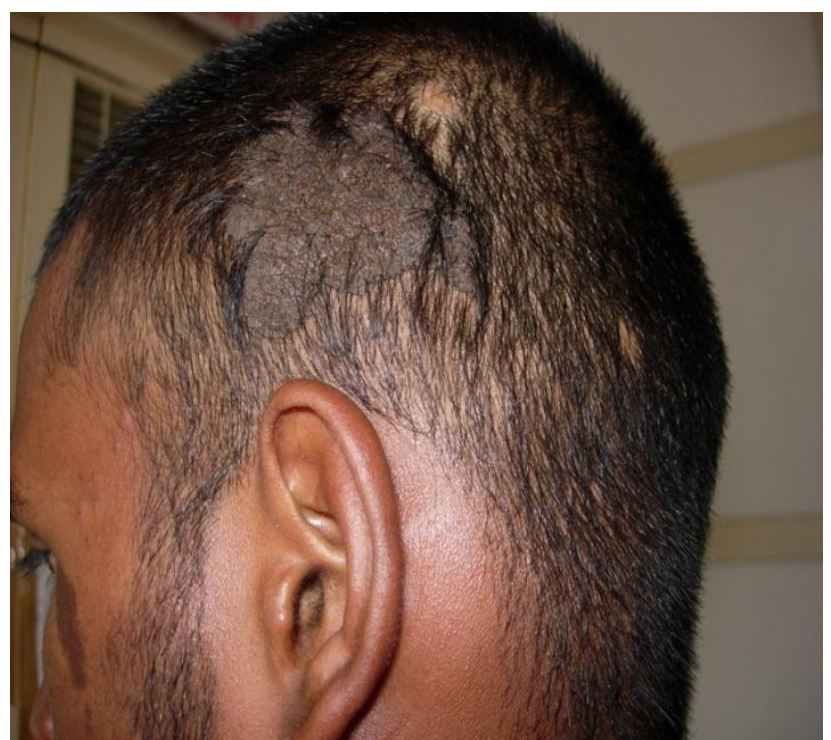

Figure 2: Nevus Sebaceous

\section{Trichoepithelioma}

Three cases of trichoepithelioma were encountered. All were adults. Of whom 2 were male and 1 female. The lesions were seen as raised, rounded, skin colored, firm papules and nodules of size varying from 2 to $8 \mathrm{mms}$ in diameter, with few showing telangiectasia over the surface. They were located on the face especially over the paranasal areas, forehead and cheeks. One of these patients underwent plastic surgical repair with good cosmetically results. All the patients reported only for cosmetic disability. Excision biopsy of a lesion was done and histopathology with hematoxylin \& eosin stain showed multiple horn cysts and islands of basaloid or basalioma cells as in the, which lack high grade, atypia and mitotic activity. Cosmetic surgical repair is the best mode of therapy.

\section{Steatocystoma Multiplex.}

Two adult male patients with steatocystoma multiplex were seen during the study period. Clinically, the lesions appeared as numerous, small, smooth, round, soft to firm, yellowish cystic nodules of size varying from 1 to $3 \mathrm{cms}$ in diameter, adherent to the overlying skin and were found predominantly over the chest and neck. The lesions, to start with were single and in course of few months, new lesions appeared. On puncturing the cysts, oily fluid was extruded in both the cases. There were no other associated skin lesions in both the patients. Excision biopsy of single lesion was done and histopathology with hematoxylin \& eosin stain showed intricately folded cyst in the dermis and numerous sebaceous gland lobules attached to both sides of the cyst wall contents of the cyst were eosinophilic.

\section{Basal Cell Epithelioma}

Five cases were encountered during the period of study, of which 3 were males $(60 \%)$ and 2 were females $(40 \%)$. The age and sex distribution in these patients is depicted in the Table- 5.Of these 5 patients 4 had nodulo-ulcerative and one had pigmented type. All the patients had the lesions of basal cell carcinoma in the face mainly over the cheeks, nose and the pre-auricular region. Biopsy was done and histopathology with hematoxylin \& eosin stain showed tumour masses of basaloid cells and horn cysts with peripheral pallisading and clefts around the islands of basalioma cells with peritumoural lacuna. All the patients were advised surgical intervention with complete excision followed by cosmetic reconstruction in the plastic surgery department. The patients were followed up only for a short period of time (2-6) months during the study and were advised further follow up every 6 months- 1 year.

\section{Squamous Cell Carcinoma}

One male and two female cases were reported during the study. Sites involved were lower lip, oral mucosa and one case was associated with xeroderma pigmentosum (Figure3) .Other case of squamous cell carcinoma was associated with growth retardation and hypogonadism. Incision biopsy of the lesion was done and histopathology with hematoxylin \& eosin stain showed massive hyperplasia and horn pearls. 


\section{International Journal of Science and Research (IJSR) \\ ISSN (Online): 2319-7064}

Index Copernicus Value (2013): 6.14 | Impact Factor (2014): 5.611

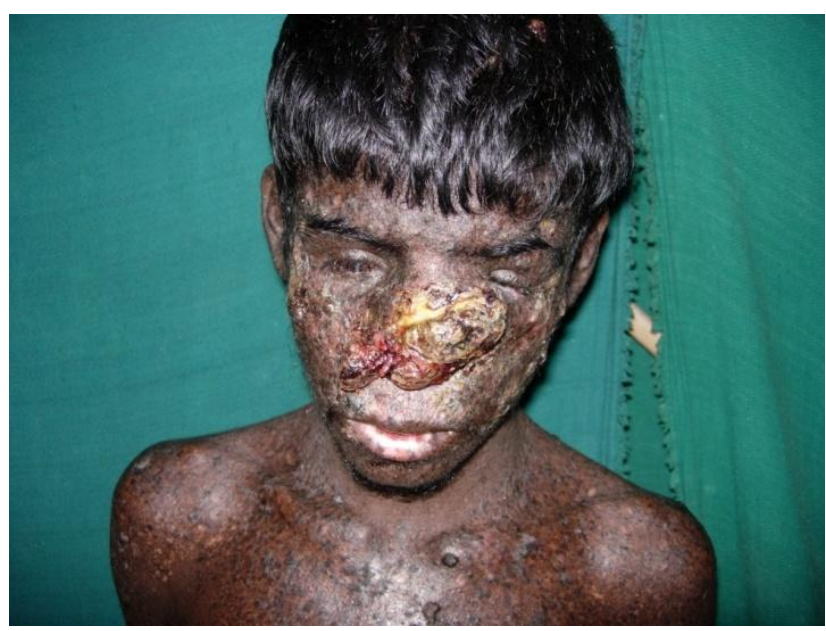

Figure 3: Squamous cell carcinoma in Xeroderma pigmentosa patient

\section{Sebaceous Carcinoma}

Only one case of sebaceous carcinoma in an albiniod female was reported in our study. The tumour has been reported in patients with arsenical intake and following radio-dermatitis and in Muir-Torre syndrome. No such associations were seen in our case. Clinically the lesion started as a plaque before plastic repair gradually increased in size, then it ulcerated. Initially we thought of basal cell carcinoma but the histopathology showed the undifferentiated cells with the centrally placed nuclei and they were grouped in a multi lobular configuration. The differentiated cells were placed centrally. Mitotic activity was seen. It was consistent with sebaceous carcinoma. Patient was referred to plastic surgery and excision repair was done.

\section{Discussion}

The incidence of patients with skin tumors (ST) reporting to the dermatology department is becoming more frequent in day-to-day practice. The study included only those patients who were complaining of their skin lesions .Almost all these sixty patients in our study sought medical help mainly for cosmetic reasons. Of these 60 patients selected for the study, females outnumbered the males.

\section{Syringoma}

Majority of the cases $(20 \%)$ in the study were having syringoma and this was the commonest tumour encountered in the study. None of these patients had familial predisposition. Most of these patients were in the adult age group and were females $(83.33 \%)$ as reported. A review of 29 cases of syringoma by Patrizi et al revealed 18 cases of eruptive syringoma, but in our study no cases of eruptive syringoma was reported [3]. The lesions were classical in their morphology, distribution and histology in all the 12 cases. No other special types such as chondroid syringoma were encountered.

\section{Seborrhoeic Keratosis}

These were second more common tumour $(16.66 \%)$ in our study. Study of Yeatman JM et al reported $100 \%$ of cases occurred in the age group over 50 years [4]. In this study also, all the cases were reported above 50 years of age. Cosmetic disfigurement was the main complaint in all the cases. One elderly female who had multiple seborrhoeic keratoses with pruritus was evaluated for possibility of Leser- Trelat sign but it did not reveal any internal malignancy. The lesions of seborrhoeic keratoses were classical in their morphology, distribution and histopathology in all the cases. Electrocautery was considered as the choice of therapy.

\section{Epidermal Nevus}

$15 \%$ of the study cases had epidermal nevus. The age of occurrence was at birth or little later as seen in these patients. These lesions were classical in their morphology, distribution and histology in all the patients. A study conducted by Vidaurida et al, reported $7.9 \%$ of epidermal nevus syndrome in 443 patients with epidermal nevi [5].But in this study one case of epidermal nevus syndrome was reported. The adult patients were encouraged to undergo surgical excision, which the best mode of therapy.

\section{Epidermal Cyst}

Epidermal cysts were $15 \%$ in our study cases. A clinicopathological study conducted by ChandrasekaranV et al reported that epidermal cysts had punctum in $40 \%$ of the cases. In this study, punctum was seen in $44 \%$ of the cases [6]. All these patients were asymptomatic excepting for the cosmetic impairment. The lesions were classical in their morphology and histology. Cosmetically a good response was observed in all the cases following complete excision of the cysts.

\section{Keratoacanthoma}

These were sixth in order $(5 \%)$ in our study cases. No precipitating factors like contact with tar and mineral oil was associated. All these were solitary lesions. All these were classical in their morphology, distribution and histopathology. None of these developed into malignancy in the follow-up. One case of keratoacanthoma occurred over the site of previous trauma over the left index finger was noted as in the study conducted by Sean et al [7]. Excision biopsy was advised for these cases to rule out squamous cell carcinoma.

\section{Nevus Sebaceous}

The incidence of nevus sebaceous was $5 \%$ in our study. Clinico pathological study of nevus sebaceous of 104 cases by Chang Geng et al, reported most of the cases developed before 10 years $(83 \%)$ as opposed to all the cases presented at birth in this study [8].. The lesions were classical in their morphology, distribution and histology in all the cases. None of the patients had any systemic associations like skeletal or neurological defects as a part of neuro-cutaneous syndrome or malignant transformation in the lesions was noted. Cosmetically acceptable surgical removal was carried out in all the cases .In general, all the patients with these lesions are better encouraged to undergo wide excision of these lesions, even at the early age to prevent the rapid progression of lesions at puberty and possible malignant transformation.

\section{Trichoepithelioma}

Three cases $(5 \%)$ were reported in our study. Cosmetically trichoepithelioma were more disfiguring than syringoma. The familial occurrences in $50 \%$ of the patients also 


\section{International Journal of Science and Research (IJSR) \\ ISSN (Online): 2319-7064}

Index Copernicus Value (2013): 6.14 | Impact Factor (2014): 5.611

prompted us for giving genetic counseling for them. Ziprkowski et al reported familial occurrence of trichoepithelioma in $50 \%$ of the patients [9]. The lesions in all the cases were classical in their morphology, distribution and histopathology. Neither the special types such as giant or desmoplastic nor ulcerative and malignant transformation were encountered in any of the cases.

\section{Steatocystoma Multiplex}

The incidence of steatocystoma multiplex was $3.33 \%$ in our study cases. Even though this disorder is dominantly inherited, our patients did not give family history as in the study conducted by Chas et al at which reported most of the cases were sporadic [10]. The lesions were classical in their morphology, distribution, and histopathology in all the cases. Puncturing the lesions extruded oily fluid which helped to diagnose the condition clinically. There were no associated skin lesions like lichen planus, pachyonchia congenita in these cases.

\section{Basal Cell Epithelioma}

Five cases of basal cell carcinoma $(8.33 \%)$ were reported in our study cases. Almost all the patients were asymptomatic except for their cosmetic disability. Prolonged exposure to sunlight could be the cause for the tumour in two of our patients who were manual laborers. A study by Christensen LJ et al reported that nodular basal cell carcinoma was the most common subtype [11] .In this study also; nodular basal cell carcinoma was the common type. The adult onset, clinical features and histopathology coincided with the literature reports. None of the other types of basal cell carcinoma like superficial, fibrosing, fibro-epithelioma types were encountered. A good response was observed in all these patients who were motivated to undergo surgical excision considering the local invasive nature of the tumour.

\section{Squamous Cell Carcinoma}

A study by Deo SV et al on the skin tumors had $55.8 \%$ squamous cell carcinoma [2], whereas in our study, squamous cell carcinoma was only $5 \%$. One of case was associated with xeroderma pigmentosum as shown in the figure. In one other case, squamous cell carcinoma in 21 years old was associated with physical growth retardation and hypogonadism. The intelligence was normal. In that case squamous cell carcinoma presented in the left angle of the mouth involving the mucosa. Clinically it presented as ulcers and histopathology was consistent with squamous cell carcinoma.

\section{Sebaceous Carcinoma}

Kumaraswamy et al reported the incidence of $17.7 \%$ of sebaceous carcinoma as against $1.66 \%$ of seborrhoeic carcinoma in this study [12]. It presented as a plaque in an albiniod female in the right cheek and later it ulcerated over the face which is common site for this tumour and with characteristic histopathology. This patient was motivated for plastic surgery for excision.

\section{Conclusion}

In India, an overall incidence of benign skin tumors is more than malignant ones. Most of the malignant tumors occur in older age group. Majority of the patients reported in this study were adults $(80 \%)$. The tumors such as epidermal nevus, nevus sebaceous occur in childhood $(20 \%)$ which insists the awareness among the parents to bring the children at an early age. The reporting of the skin tumors was more by the females $(51.66 \%)$ compared to males $(48.33 \%)$ probably because of their cosmetic awareness. The commonest benign tumour encountered in this study was syringoma $(20 \%)$ which were mainly in the infra-orbital region. The second common tumour was seborrhoeic keratosis $(16.66 \%)$. Among the malignant tumor, basal cell carcinoma $(8.33 \%)$ is most common. Rare presentations like squamous cell carcinoma with growth retardation and sebaceous carcinoma in an albinoid female has been documented in this study. More studies are needed to ascertain the frequency of the skin tumors.

\section{References}

[1] Kupfer.T.Epidermal cytokines. The immunopathology of cells, cytokines, Oxford university press, 1988.

[2] Deo S.V., Hazarika S., Shukla N., Kumar S., Kar M., Somaiya A. - Surgical management of skin cancers: Experience from a regional cancer centre in North India. Ind J Cancer. 42:145-150, 2005.

[3] Kikuchi I et al; Plaque type Syringoma; J Derm 1979, 6,329-31

[4] Yeatmen jm et al; The prevalence of Seborrhoeic keratosis in and their Frequencey.Br jd 137, 411, 1997.

[5] Vidaurri-de la Cruz $\mathrm{H}^{1}$, Epidermal nevus syndromes: clinical findings in 35 patients. Pediatric Dermatol 2004 July 21 (4);432-9.

[6] V. Chandrasekaran, Epidermal cysts - a clinicopathological and biochemical study. Postgrad Med J 1980;56:823-827 doi:10.1136/pgmj.56.662.823.

[7] Pattee, Sean F. et al. Keratoacanthoma developing in sites of previous trauma: A report of two cases and review of the literature. Journal of the American Academy of Dermatology, Volume 48 , Issue 2, S35 S38.

[8] Chen MJ. [Nevus sebaceus--a clinicopathological study of 104 cases]. Changgeng Yi Xue Za Zhi. 1990 Sep;13(3):199-207.

[9] Ziprowski L, Sehewach - Millet M. Multiple trichoepitheliomas in a mother and two children. Dermatologica. 1966; 132: 248-256.

[10] Cho S, Clinical and histologic features of 64 cases of steatocystoma multiplex. J Dermatol. 2002 Mar;29(3):152-6.

[11] Christenson $\mathrm{LJ}^{1}$, Incidence of basal cell and squamous cell carcinomas in a population younger than 40 years. JAMA. 2005 Aug 10;294(6):681-90.

[12]Reddy MK. A clinicopathological study of adnexal tumours of skin. Indian J Med Res. 1982 Jun;75:882-9. 